SESSION IV. BINARIES - Chair: Anthony F.J. Moffat 


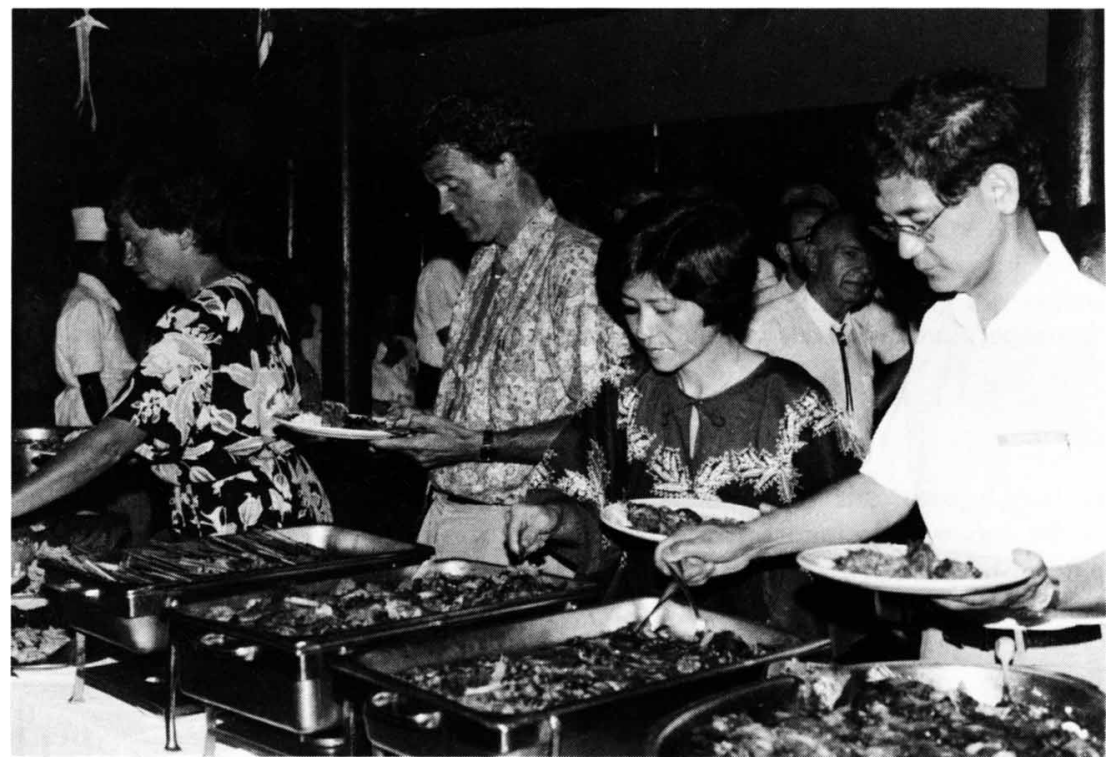

Mrs. Moffat, Tony Moffat, Mrs. Nomoto, Ken Nomoto 


\title{
WOLF-RAYET BINARIES: OBSERVATIONAL ASPECTS
}

\author{
A.M. Cherepashchuk \\ Sternberg Astronomical Institute \\ Moscow University \\ 119899, Universitetsky Prospect \\ 13, Moscow, USSR
}

\begin{abstract}
New data concerning observational aspects of Wolf-Rayet binaries are summarised. $W R+O$ and $W R+c$ binary systems are considered. All the data concerning WR binaries agree well with the modern understanding of WR stars as the helium remnants formed as a result of mass loss by massive 0 -type stars.
\end{abstract}

\section{Introduction}

The problem of Wolf-Rayet (WR) binaries consists of two parts: WR+O systems and $\mathrm{WR}+\mathrm{c}$ systems. In the latter, relativistic objects (neutron star or black hole) are presumed to exist, mainly on the basis of modern evolutionary considerations.

\section{Parameters of WR+O binary systems}

The fraction of WR+O binary systems among all WR stars in the Galaxy is of the order of $43 \%$ (Moffat et al., 1986). This value is close to the fraction of binary systems among O-stars (Abbott and Conti, 1987). One of the important criteria for duplicity of WR stars is the enhanced X-ray radiation which originates near the front of the shock wave formed near the O-star by the supersonic flow of the WR wind (Cherepashchuk, 1976). At present several new WR+O binaries have been identified using this criterion (Pollock, 1987). In this connection the fraction of WR+O binaries among WR stars is compatible with the above $43 \%$.

A comparison of the galactic distribution of WR+O systems and single WR stars is given by Hidayat et al. (1984), Tutukov and Yungelsen (1985), Doom (1987), van der Hucht et al. (1988), and Conti and Vacca (1990). In the galactic plane, the increase of the fraction of binary WR stars with increasing galactocentric distance is observed. For $\mathrm{d} \leq 4 \mathrm{kpc}$ $<|z|>($ single WR $)=48 \mathrm{pc},<|z|>(\mathrm{SB} 2+\mathrm{mSB} 1)=59 \mathrm{pc},<|z|>(\operatorname{lm~SB} 1)=134 \mathrm{pc}($ see however, Conti and Vacca, 1990). All these facts agree well with the evolutionary scenario for massive close binary systems (Tutukov and Yungelson, 1973, van den Heuvel, 1976). The system RY Sct may be considered as the progenitor of a WR+O binary (Antokhina and Cherepashchuk, 1988a).

The fraction of binaries among WR stars in other galaxies varies from $40 \%$ for the LMC to $\geq 70 \%$ for the SMC (Moffat, 1988). One should note the absence of SB2 binaries among WN8, 9 stars (Moffat, 1989). 
The characteristics of well-studied WR $+\mathrm{O}$ binaries are listed, e.g., by Smith and Maeder (1989). Many masses are calculated using values of the inclination of the orbital plane $i$ determined from light curves and from polarisation curves. The basic conclusions concerning the properties of $\mathrm{WR}+\mathrm{O}$ binaries are the following.

2.1 The mass ratio $q=\frac{m_{W R}}{m_{O}}$ for $\mathrm{WR}+0$ binaries falls in the range $0.17-2.67$. The mean value is $q=0.44-0.53$ (here and below), the second value comes from including the system HD92740 with $q \simeq 2.67$ ). The dependence of $q$ on spectral subtype Sp is presented in Fig. 1. This correlation was pointed out by Moffat (1982) and Moffat et al. (1990). In particular, the continuous decrease of mass ratio $q=\frac{m_{W R}}{m_{0}}$ with hotter WN and especially WC subtypes is observed. For WC subtypes the value of $q$ ranges from $\sim 0.5$ for WC8 to $\sim 0.2$ for WC4-WO4. For WN subtypes, the value of $q$ ranges from 2.67 to $0.2-0.3$ for WN7 to WN3, respectively. As was pointed out by Moffat et al. (1990), these facts support the idea of evolution of WR stars through the sequence WN-WC8-WC4-WO.

Fig. 1

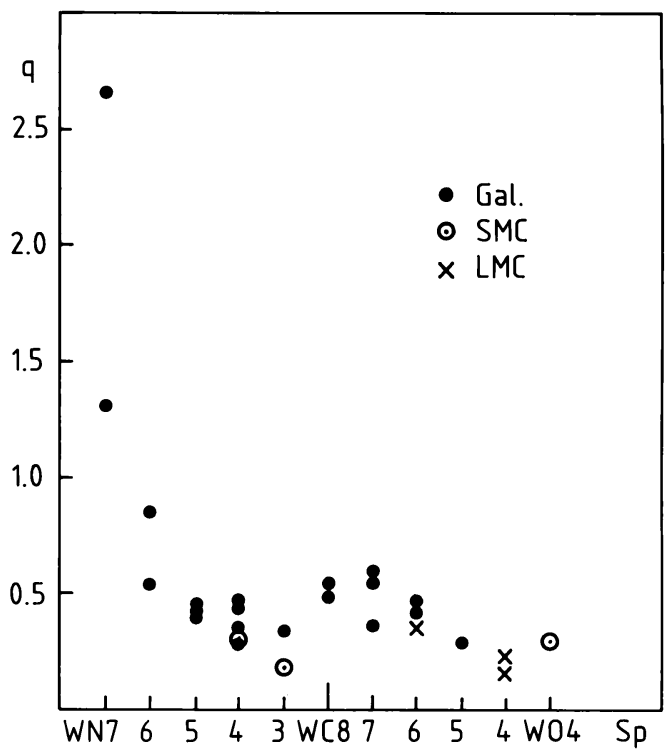

2.2 The mean mass of WR stars (22 determinations) is $15.6-18.4 M_{\odot}$. The range of masses of WR stars is very wide: from $5 M_{\odot}$ to $48 M_{\odot}$ and even $77 M \odot \odot$ (HD92740). The mean mass of WN stars is $17.5-22.5 M_{\odot}(12$ stars $)$ and ranges from 8 to $48 M_{\odot}$ and $77 M_{\odot}$. The mean mass of WC stars (9 stars) is $13.4 M_{\odot}$ and ranges from $5 M_{\odot}$ to $27 M_{\odot}$. The mass of the WO4 star is $14 M_{\odot}$. Therefore, recent data allow one to suggest that the masses of WC stars on the average are less than those of WN stars. This is in agreement with the modern evolutionary scenario for WR stars. The dependence of the masses of WR stars on their spectral subtypes and the correlation between the masses of WR stars and those of $\mathrm{O}$-stars for WR+O systems are presented in Fig. 2.

2.3 The masses of the 0 -companions in WR+O binaries range from 14 to $57 M_{\odot}$ and on average have the value $32.8 M_{\odot}$. The mean mass for $\mathrm{O}$-stars in $\mathrm{WN}+\mathrm{O}$ systems is 
Fig. 2
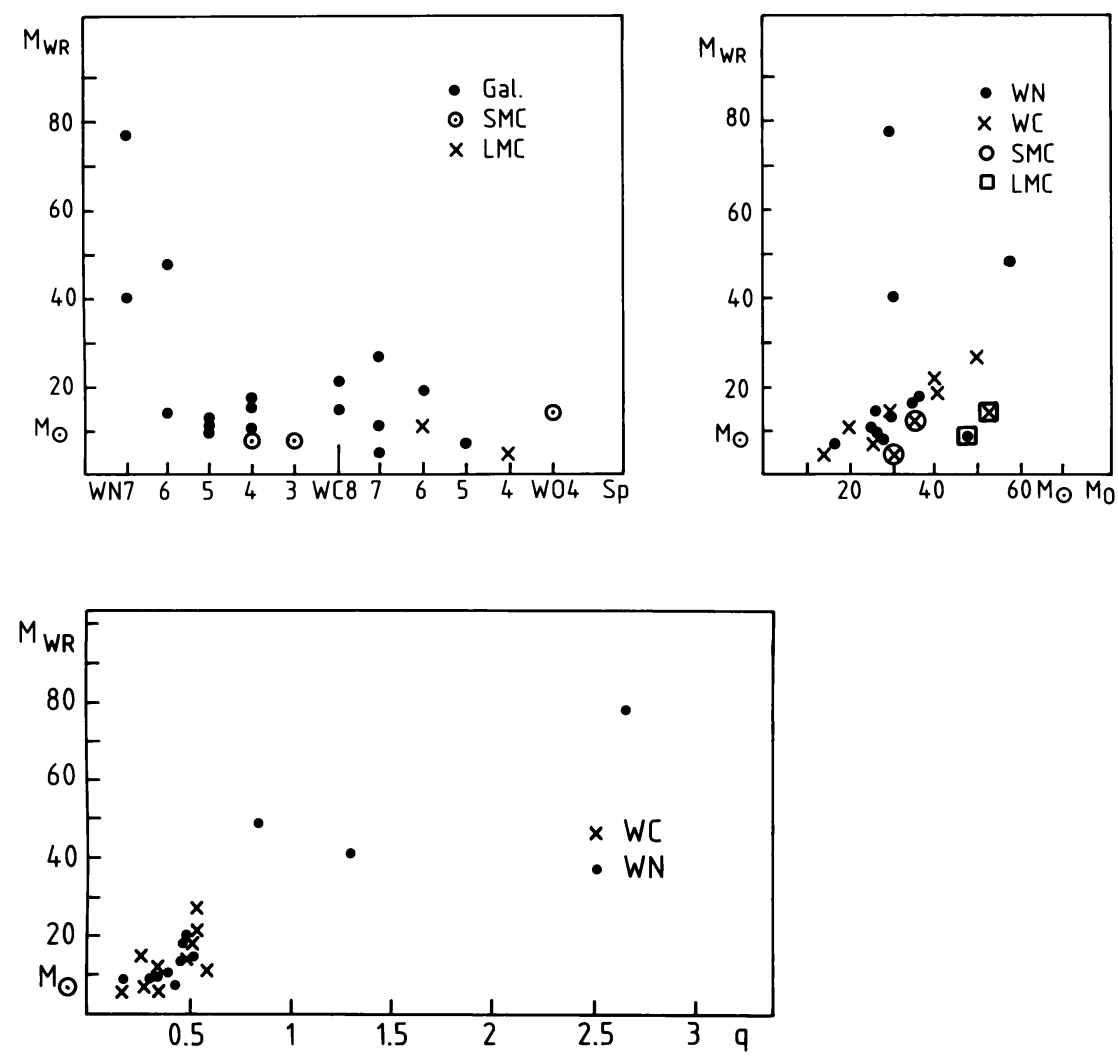

Fig. 3

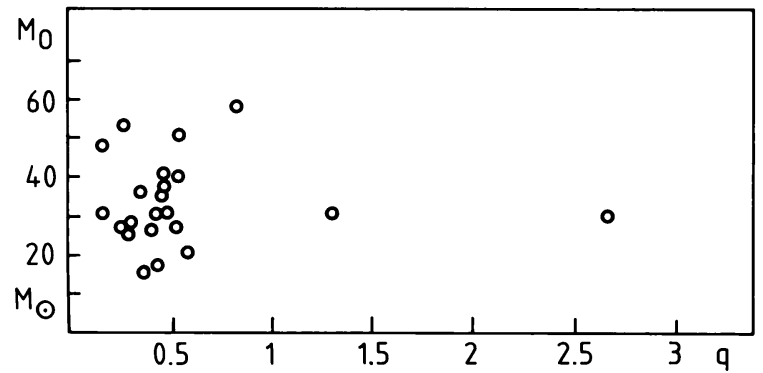

$31.2 M_{\odot}$ and for 0 -stars in $\mathrm{WC}+\mathrm{O}$ systems is $32.4 M_{\odot}$. The mass of the 0 -companion in the W04+04V system $\mathrm{AB} 8$ is $52 M_{\odot}$.

2.4 There is a good correlation between the mass ratio $q$ and the mass of WR stars in WR+O systems (Fig. 3): the mass of the WR star increases with q. Between the mass of the 0-companion and $q$ there is no clear correlation (Fig. 3 ).

2.5 The mean value of the total mass of the $W R+0$ systems is $48.5-51.1 M_{\odot}$ and falls 
in the range $19-106 M_{\odot}$. The mean value of the total mass for the $\mathrm{WN}+\mathrm{O}$ systems is $50-54.7 M_{\odot}$; for the WC+O systems it is $44.6 M_{\odot}$; and for the WO+O system it is $66 M_{\odot}$. These values are almost equal to the mean total mass for $0+O$ systems $\sim 46 M_{\odot}$ (see, e.g., Svechnikov, 1989). The total mass for $W R+0$ systems seems to increase with $q$; this is not observed for $\mathrm{O}+\mathrm{O}$ systems. The total mass for $\mathrm{WR}+\mathrm{O}$ systems shows a slow decrease with the spectral subtype of the WR component: from $\sim 85 M_{\odot}$ for WN7+O to $\sim 50 M_{\odot}$ for (WC4-WO4) + $\mathrm{O}$ systems. The $\mathrm{O}$-companions of $\mathrm{WR}+\mathrm{O}$ binary systems show a large scatter in mass; on average their masses are independent of the spectral subtype of the WR-companion.

2.6 Values of eccentricities of orbits for WR+O systems with periods $\geq 70$ days are $e=$ $0.3-0.8$. All the systems with periods $P \leq 14$ days have circular orbits $(e=0)$. In the interval $14^{\mathrm{d}}<P<70^{\mathrm{d}}$ there are circular and eccentric orbits $(e=0.17-0.5)$.

2.7 The bremsstrahlung X-ray luminosity in WR+O binaries (Pollock, 1987) is correlated with orbital period: $L_{x}$ is greater for systems with shorter periods. In $\mathrm{WN}+\mathrm{O}$ systems, the mean luminosity $\bar{L}_{x}=16 \times 10^{32} \mathrm{erg} / \mathrm{s}$ for $P<20^{\mathrm{d}}$ and $\bar{L}_{x}=10 \times 10^{32} \mathrm{erg} / \mathrm{s}$ for $P \geq 20^{\mathrm{d}} ;$ for WC+O systems $\bar{L}_{x}=12 \times 10^{32} \mathrm{erg} / \mathrm{s}$ for $P<20^{\mathrm{d}}$ and $\bar{L}_{x}=1.5 \times 10^{32} \mathrm{erg} / \mathrm{s}$ for $P \geq 20^{d}$. This correlation is consistent with the theory for the generation of $\mathrm{X}$-rays in the stellar winds of WC stars, which are enriched by heavy elements of the CNO group because of the more advanced evolutionary stage of WC stars.

\section{X-ray radiation from WR+O binary systems}

Cherepashchuk (1967) pointed out that considerable X-ray radiation in WR+O binary systems may be generated in the shocked region formed in front of the $\mathrm{O}$-star by the supersonic wind of the WR companion. This idea was developed by Prilutsky and Usov (1976). Calculations carried out for realistic WR+O systems, taking into account the Compton cooling of hot plasma by optical radiation of $\mathrm{O}$ and WR stars (Cherepashchuk, 1976), have shown that the expected X-ray luminosity in the majority of cases is $10^{32}-10^{34}$ $\mathrm{erg} / \mathrm{s}$ in the mean energy range $\mathrm{kT}=1-10 \mathrm{keV}$ for mass loss rates from the WR star $\dot{\mathrm{M}}$ $=10^{-6}-10^{-5} M_{\odot} / \mathrm{yr}$, respectively. Further development of the theory (Bayramov et al., 1988) has confirmed these results.

Recent results on the analysis of EINSTEIN X-ray observations of 48 WR stars (Pollock, 1987 ) in the range $0.2-4 \mathrm{keV}$ have shown that the X-ray luminosity for WR stars falls in the range $L_{x}=10^{31}-10^{34} \mathrm{erg} / \mathrm{s}$. The mean X-ray luminosity of single WR stars is $\sim 5 \times 10^{31} \mathrm{erg} / \mathrm{s}$; however, the X-ray luminosity of WR+O binaries is one to two orders of magnitude higher. The orbital phase-dependent variability of the intensity and "hardness" of the X-ray radiation have been observed in several cases of WR+O binaries (Pollock, 1987; Moffat et al., 1982). Using ultraviolet IUE observations, the effects of the colliding stellar winds have been discovered recently in the V444 Cyg system by Shore and Brown (1988). Thus, the prediction of shock waves and considerable X-ray radiation in binary WR +O systems (Cherepashchuk, 1967, 1976; Prilutsky and Usov, 1976) is now confirmed by new X-ray and UV observations.

There is some discrepancy between the $\mathrm{X}$-ray observations and the theory: the mean observed X-ray luminosity for WR+O binary systems is more than one order of magnitude lower than the theoretical luminosity, and the mean hardness of the X-ray spectrum is kT $\sim 1 \mathrm{keV}$ instead of the theoretically predicted value of $\mathrm{kT} \sim$ several $\mathrm{keV}$. It was shown recently (Cherepashchuk, 1990), that the relatively low X-ray luminosity of WR+O binaries may be connected with the ragged, cloudy structure of WR winds (Cherepashchuk et al., 
1984, van Genderen et al., 1987, Moffat et al., 1988, Antokhin et al., 1988). Further X-ray observations of $\mathrm{WR}+\mathrm{O}$ binaries, investigations of the regular orbital variability of their $\mathrm{X}$-ray luminosity and the fluctuations of X-ray flux on a timescale of about $10^{3} \mathrm{~s}$ would be very important for the determination of the structure and dynamics of stellar WR winds and their chemical composition.

\section{Variable linear polarisation of optical radiation from $W R+O$ binary systems}

The first discovery of variable linear polarisation in a WR+O system (V444 Cyg) was found in V444 Cyg (Rudy and Kemp, 1978). A major effort to expand polarimetric investigations of WR+O binaries and WR stars in general has been undertaken by Moffat and collaborators (Moffat, 1988; Robert and Moffat, 1989; Robert et al., 1989; Drissen et al., 1987; St.-Louis et al., 1988). Up to now, polarimetric determinations of $i$ and characteristics of the instability of WR winds have been determined for about two dozen WR+O binaries (e.g. St.-Louis et al., 1988). For the system V444 Cyg, polarimetric data yield $i=78.7^{\circ} \pm 0.5^{\circ}$ and WR core radius $r_{c}^{W R}=3 R_{\odot}$ (Robert et al., 1989; Moffat, 1989) which are in good agreement with light-curve solutions (Cherepashchuk, 1975; Cherepashchuk et al., 1984). In the recent work of Hamann et al. (1990), who have made significant progress in modelling Non-LTE extended atmospheres, a new attempt to determine the parameters of V444 Cyg has been carried out. Taking into account the observed spectroscopic estimate of the luminosity of the WN5 star $L_{W}=0.167-0.254$ (Beals, 1944; Annuk, 1988) we are obliged to choose the light curve solution from the work of Hamann et al. (1990) corresponding to $r_{c}^{W R} \geq 1.4 R_{\odot}$ and $T_{c} \geq 60000 \mathrm{~K}$. This result is close to our solution for the light curve of the V444 Cyg system (Cherepashchuk, 1975; Cherepashchuk et al., 1984): core radius and core temperature for the WN5 star: $r_{c}^{W R}=2.6-2.9 R_{\odot}, T_{c}=70000-100000$ K.

The parameters of V444 Cyg proposed by Underhill and Fahey (1987) (in particular $\left.r_{c}^{W R}=9 R_{\odot}\right)$ strongly disagree with the continuum light curve at $\lambda 4244 \AA$. Third light proposed by Underhill and Fahey (1987) for the UV spectral range is negligible in the optical at the $\lambda 4244$ light curve because variations at $\lambda 4244$ between the minima are small $\left(\sim 0^{\mathrm{m}} .01\right)$.

Up to now, the accuracy of the light curve of the new WR+O eclipsing binary HD5980 is not sufficient for a unique light curve solution. Further observations of HD5980 are needed.

St.-Louis et al. (1988) have confirmed on average the increase of $\dot{M}$ with mass $M$ of WR stars from polarimetric data. According to Moffat et al. (1988), the WNL stars, with smaller values of wind velocity, exhibit greater amplitudes of random fluctuations in linear polarisation. This may be connected with instabilities in the WR star wind leading to the formation of dense blobs of matter.

\section{Ultraviolet IUE observations of WR+O systems; selective atmospheric eclipses}

The effect of enhanced opacity in the stellar WR wind has been discovered by Eaton et al. $(1982,1985 \mathrm{a}, \mathrm{b})$ for the ultraviolet region of the spectrum at $\lambda<2000 \mathrm{~A}$. This effect is observed in the eclipsing WR+O binaries V444 Cyg and CV Ser (Eaton et al., 1985a,b; Koenigsberger and Auer, 1985) and in several other spectroscopic binaries (see e.g. Hutchings and Massey, 1983). The depth of the atmospheric eclipse strongly increases in the range $\lambda<1500 \mathrm{~A}$; this is caused by the absorption of $\mathrm{O}$-star light in the many lines of ions of FeIV, FeV, and FeVI (Koenigsberger, 1988). In the atmospheric eclipse of HD5980, 
Koenigsberger et al. (1987) did not find the increased absorption at $\lambda \lambda 1300-1500 \mathrm{~A}$; this reflects the relatively poor abundance of heavy elements in the SMC.

A theory of selective atmospheric eclipses in WR+O binary systems has been developed by Khaliullin and Cherepashchuk (1976). For the first time, selective atmospheric eclipses were discovered in the WR+O system CV Ser (Cherepashchuk, 1969,1971) in the emission band CIII-CIV $4653 \AA$.

Underhill et al. (1988) have criticized our model of selective atmospheric eclipses for WR+O binaries. The considerations of Underhill et al. (1988) are fully true for the absolute intensity of an absorption line arising in the spectrum of the $\mathbf{O}$ star during atmospheric eclipse in V444 Cyg. But their final conclusion is incorrect because Underhill et al. did not take into account the fact that this absorption is veiled by strong, non-eclipsed emission line flux, formed in the WR envelope.

\section{New investigations of some eclipsing WR+O binary systems}

\subsection{THE V444 Cyg SYSTEM}

The parameters of this system determined from the light curve solutions (Cherepashchuk, 1975; Cherepashchuk et al., 1984) and from the polarimetric analysis (Robert et al., 1989) seem to be rather reliable: $r_{O 6}=10 R_{\odot}, i=78^{\circ}, L_{W} \simeq 0.2, r_{c}^{W R} \leq 2.9 R_{\odot}, T_{c}^{W R}>60000 \mathrm{~K}$. The structure of the extended atmosphere of the WN5 star from the analysis of multicolor light curves at $\lambda \lambda 2460 \AA-3.5 \mu$ has been determined by Cherepashchuk et al. (1984). The results concerning V444 Cyg have been used by Pauldrach et al. (1985) for the calculation of the model of the WR star wind driven by radiation. Results of the interpretation of the light curve of V444 Cyg have also been used by de Greve and Doom (1988) for a model calculation of the origin and evolution of the V444 Cyg binary. Theoretical analysis of the WN5 companion in the V444 Cyg system has been done by Vanbeveren (1988) and Poe et al. (1989). New estimates of the mass loss rate from the WN5 star in V444 Cyg on the basis of orbital period change are: $(1.1 \pm 0.2) \times 10^{-5} M_{\odot} / \mathrm{yr}$ (Khaliullin et al., 1984) and $0.6 \times 10^{-5} M_{\odot} / \mathrm{yr}$ (Underhill et al., 1990). New spectroscopic elements of the orbit for V444 Cyg have been determined by Underhill et al. (1988a) and Acker et al. (1989). Rapid oscillations in the intensity of the emission line HeII 4686 in V444 Cyg have been discovered by Zilyaev and Marchenko (1988).

\subsection{THE CQ Cep SYSTEM}

Analysis of $I U E$ ultraviolet spectra (e.g. using new determinations of radial velocities from archive spectra) and UBVJKL photoelectric observations of CQ Cep have been made by Stickland et al. (1984). It is shown that CQ Cep belongs to the Cep OB1 association $(\mathrm{d} \simeq$ $\left.3.5 \mathrm{kpc}, \mathrm{A}_{\mathrm{V}}=2^{\mathrm{m}} .25\right)$. A photometric solution of the light curves of CQ Cep has been carried out by Leung et al. $(1983): q=1.33, M_{W N 7}=46 M_{\odot}, M_{O}=35 M_{\odot}, i=68^{\circ}, T_{c}^{W R}=45000$ $\mathrm{K}$ (adopted), $T_{O}=41000-42000 \mathrm{~K}$. Polarimetric investigations (Drissen et al., 1986) yield: $i=78^{\circ}, M_{W N 7}=42 M_{\odot}, M_{O}=30 M_{\odot}$. New spectroscopic and photometric observations are also available: Kartashova and Snezh ko (1985), Shylaja (1986), Harvig (1987), Underhill et al. (1990). The decreasing of the orbital period of CQ Cep is confirmed by Antokhina et al. (1987): $\dot{P}=-0.014 \pm 0.004 \mathrm{~s} / \mathrm{yr}$. A new photometric solution of the light curves of CQ Cep, using new information about its distance (Stickland et al., 1984), has been obtained by Antokhina and Cherepashchuk (1988b): $i=77^{\odot}, q=1.3-1.6, M_{W N 7}=$ $(42-66) M_{\odot}, M_{O}=(32-41) M_{\odot}, r_{O}=(9.4-10.1) R_{\odot}, T_{O}=(40000-50000) K, r_{c}^{W N 7}=$ 
$(10.6-12.5) R_{\odot}, T_{c}^{W N 7}=(60000-70000) \mathrm{K}$. If $d<3.5 \mathrm{kpc}$, the values of the masses and temperatures of the components will be smaller. These results are in agreement with those of Lipunova and Cherepashchuk (1982). Other parameters of CQ Cep have been proposed by Underhill et al. (1990); they are in conflict with the shape and amplitude of the light curve.

The disk-like model for WR star envelopes, proposed by Underhill (1984) and Underhill et al. $(1988 \mathrm{a}, 1990)$, disagrees with observations: up to now there is no correlation between the inclination of the orbit plane and the widths of emission lines and other parameters in WR+O binaries (Aslanov and Cherepashchuk, 1990).

\subsection{THE CV Ser SYSTEM}

A double envelope nebula structure has been discovered around CV Ser by Gonzalez and Rosado (1984).

Analysis of UV selective atmospheric eclipses in CV Ser using IUE data has been carried out by Eaton et al. (1985). The parameters of CV Ser have been determined from a light curve solution by Lipunova (1982): $i=67^{\circ} \pm 3^{\circ}, r_{c}^{W C 8}=(3.5 \pm 0.5) R_{\odot}, T_{c}^{W C 8} \geq 55000$ $\mathrm{K}$. It is of great interest to investigate the very peculiar and puzzling variability of CV Ser and the WC9 star HD 164270 on a long time scale. Both stars show considerable $\left(\sim 00^{\mathrm{m}} 5\right)$ and rather rare (once per several dozens of years) decreases in light.

\subsection{THE CX Cep SYSTEM}

Polarimetric observations of CX Cep have been carried out by Schulte-Ladbeck and van der Hucht (1989). The orbital inclination is estimated from the polarimetric data: $i=74^{\circ} \pm 5^{\circ}$; corresponding masses are $M_{W N 5}=6 M_{\odot}, M_{O 8}=14 M_{\odot}$. From the light curve solution (adopting $M_{O 8}=22 M_{\odot}$ ) the values $i=53^{\circ}$ and $M_{W N 5}=10 M_{\odot}$ were determined by Lipunova and Cherepashchuk (1982). Our attempt to interpret the light curve of CX Cep with the value $i=74^{\circ}$ was not successful.

Intense photometric observations of $\mathrm{WR}+\mathrm{O}$ binaries have been carried out by Monderen et al. (1987), Moffat and Shara (1986), Lamontagne and Moffat (1987), van Genderen et al. (1987), Balona et al. (1989).

The results of the interpretation of the light curves of $W R+O$ eclipsing binaries are in agreement with modern ideas about the nature of WR stars: WR stars are helium remnants formed during mass exchange in massive close binary systems.

\section{WR+c binaries containing possible compact companions}

Numerous intense photometric investigations of the microvariability of suspected WR+c stars have been carried out (Balona et al., 1989; Moffat and Shara, 1986; Antokhin and Cherepashchuk, 1989; van Genderen et al., 1987, 1989; Lamontagne and Moffat, 1987; Monderen et al., 1988).

Up to now, only two WR stars may be considered as genuine WR +c binaries: HD50896 and HD197406. These systems are real binaries containing a compact (possibly relativistic) object $\left(M_{c} \simeq 1 M_{\odot}\right.$ for HD50896 and $M_{c}>4 M_{\odot}$ for HD197406). Evolutionary considerations and high values of $|z|$ suggest that the companions in these binary systems are relativistic (neutron star or black hole). However, it is difficult to explain for these systems the absence of strong X-ray radiation (Cherepashchuk, 1981; Stevens and Willis, 1988). One could try to find correlations between basic characteristics of WR+c systems. No correlation is observed between the period $\mathrm{P}$ and the semiamplitude of the radial velocity 
curve K. Also, no correlation exists between the period $\mathrm{P}$ and the spectral class (Sp) of the WR star, between the amplitude of the optical variability $\Delta V$ and value of $K$. These facts testify against the intrinsic variability of these stars. Between $\Delta V$ and Sp some correlation may be suggested: $\Delta V$ reaches a maximum for late WN star types (see Fig. 4). This correlation may be considered as an argument against the binary nature of these stars: at least for some of the 17 suspected WR+c binary systems the microvariability may be connected simply either with the instability of the WR wind (Lamontagne and Moffat, 1987; Drissen et al., 1987; Moffat, 1988b), or with the rotation of star spots. The maximum $\mathrm{X}$-ray luminosity $L_{X}$ is observed for the minimum value of $\mathrm{K}$. This is not in contradiction with the binary nature of $\mathrm{WR}+\mathrm{c}$ stars.

Fig. 4

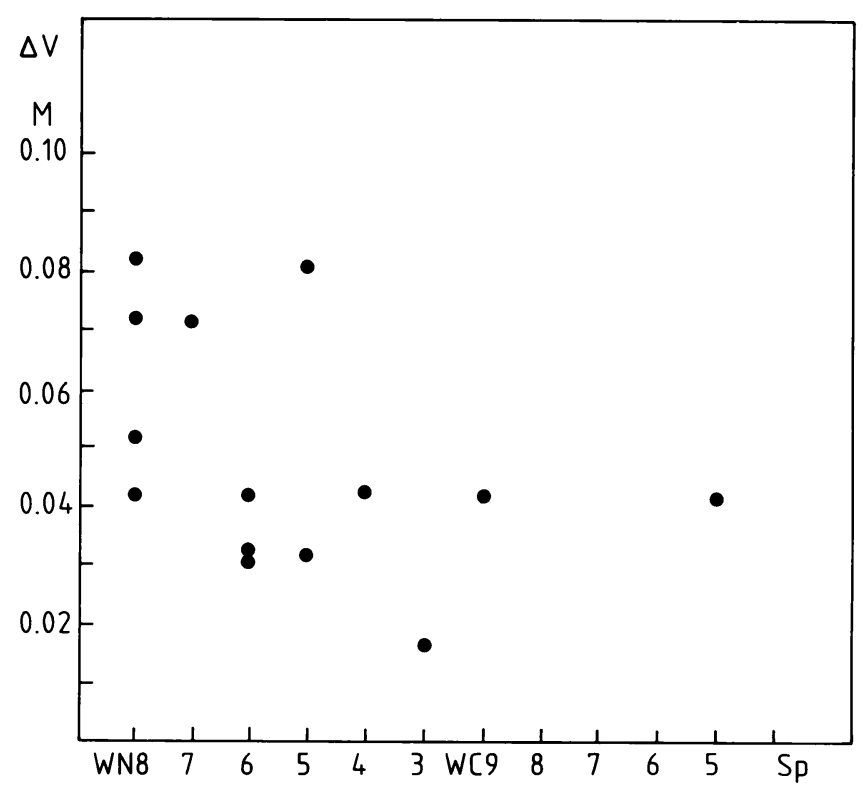

Theoretical profiles of the emission lines of WR $+c$ stars have been calculated by Antokhin (1986). The results are close to those obtained by Hatchett and McCray (1977) for X-ray binary systems. Very valuable information on the nature of WR $+c$ systems has been obtained from IUE spectroscopic data (Koenigsberger and Auer, 1987; St.-Louis et al., 1989; Smith et al., 1986). Detailed investigation of the profiles of the UV lines in the spectrum of HD1922163 (St.-Louis et al., 1989) leads the authors to the conclusion that an accreting neutron star cannot be responsible for the variability of the spectrum of HD192163. A very important effect - the decrease of the colour index of HD164270 with an increase in brightness has been discovered by van Genderen and van der Hucht (1986). This may be connected with the projection of blobs of WR wind material against the hot core of the WR star. A number of publications describing the photometric and spectroscopic variability of suspected WR+c stars as due to nonradial pulsations of single WR stars has appeared (Vreux, 1985, 1987; Gosset and Vreux, 1987). The most probable candidates 
for binary systems in the evolutionary stage after second mass exchange in massive X-ray binaries are the systems HD197406 and HD50896. As a transition case of evolution between an X-ray binary and a WR+c binary, the object SS433 may be considered (van den Heuvel, 1981; Margon, 1984; Cherepashchuk, 1988).

According to Drissen et al. (1986b) HD197406 is supposed to be an X-ray quiet black hole in a WR +c binary, with the mass of the compact object about $12.4 M_{\odot}$.

Intense photometric and polarimetric observations of HD50896 have been carried out by Lamontagne et al. (1986), Balona et al. (1989), and Drissen et al. (1989). Radial velocity variations, the linear polarisation variations and variations of $\mathrm{X}$-ray radiation (Moffat $e t$ $a l ., 1982)$ are observed with a period of $3.766 \mathrm{~d}$ for HD50896. Long-term variability of HD50896 with a cycle of about 13 years is suspected (van der Hucht et al., 1989). This may be connected with the precession of an accretion disk (Cherepashchuk, 1982). A possible supernova remnant formed after a supernova explosion in the HD50896 system has been discovered by Nichol-Bohlin and Fesen (1986). IUE and spectroscopic investigations of HD50896 have been published by Howarth and Phillips (1986) and Willis et al. (1986). Physical parameters of the extended atmosphere of the WR star HD50896 have been calculated by Hillier (1987) and Hamann et al. (1988). A rather unexpected circumstance has been noted: a genetic connection between WR+c binary systems and WR stars with enhanced oxygen lines (Barlow and Hummer, 1988). In particular, the z-distribution of the WR stars with enhanced oxygen lines seems to be quite similar to the $\mathrm{z}$-distribution for WR+c stars (Rustamov and Cherepashchuk, 1986). It should be also noted that Hogg (1989) was unable to detect radio variability of the WR+c stars HD50896, HD191765 and HD192163 with amplitudes greater than $20 \%$ on timescales from 1 to 7 years. The thermal origin of the radio emission for all these WR+c stars is confirmed.

\section{References}

Abbott, D.C. and Conti, P.S. 1987, Ann. Rev. Astron. Astrophys. 25, 113.

Annuk, K. 1988, in T. Nugis, I. Pustylnik (eds.), Wolf-Rayet Stars and Related Objects, Acad. of Sci. of Estonia, Tallinn, p. 144.

Acker, A., Prevot, M.-L., and Prevot, L. 1989, Astron. Astrophys. 226, 137.

Antokhin, I.I. 1986, Astron. Zh. 63, 1152 (=Sov. Astron. 30, 680).

Antokhin, I.I. and Cherepashuk, A.M. 1989, Pis'ma Astron. Zh. 15, 701 (=Sov. Astron. Letters 15, 303).

Antokhin, I.I., Kholtygin, A.F., and Cherepashchuk, A.M. 1988, Astron. Zh. 65, 558 (= Sov. Astron. 32, 285).

Antokhina, E.A. and Cherepashchuk, A.M. 1988a, Pis'ma Astron. Zh. 14, 252 (= Sov. Astron. Letters 14, 105).

Antokhina, E.A. and Cherepashchuk, A.M. 1988b, Astron. Zh. 65, 1016 (= Sov. Astron. 32, 531).

Antokhina, E.A., Kreiner, I.M., Tremko, I., and Cherepashchuk, A.M. 1987, Pis'ma Astron. Zh. 13, 417 (= Sov. Astron. Letters 13, 170).

Aslanov, A.A. and Cherepashchuk, A.M. 1990, Astron. Zh. 67, N6 (=Sov. Astron. .., ..).

Balona, L.A., Egan, I., and Marang, F. 1989, Monthly Notices Roy. Astron. Soc. 240, 103.

Barlow, M.J. and Hummer, D.G. 1982, in: Wolf-Rayet Stars: Observations, Physics and Evolution, IAU Symp. No. 99 (Dordrecht: Reidel), p. 387.

Bayramov, Z.T., Pilugin, N.N., and Usov, V.V. 1988, Astron. Tsirk. No. 1526, 1.

Beals, C.S. 1944, Monthly Notices Roy. Astron. Soc. 104, 205.

Cherepashchuk, A.M. 1967, Variable Stars 16, 226. 
Cherepashchuk, A.M. 1969, Astron. Tsirk. No. 509.

Cherepashchuk, A.M. 1971, Astron. Zh. 48, 1201 (=Sov. Astron. 15, 955).

Cherepashchuk, A.M. 1975, Astron. Zh. 52, 81 (= Sov. Astron. 19, 47).

Cherepashchuk, A.M. 1976, Pis'ma Astron. Zh. 2, 356 (= Sov. Astron. Letters 2, 138).

Cherepashchuk, A.M. 1981, Monthly Notices Roy. Astron. Soc. 194, 755.

Cherepashchuk, A.M. 1982, Astrophys. Space Sci. 86, 299.

Cherepashchuk, A.M. 1988, in: R.A. Sunyaev (ed.), Soviet Sci. Rev. Astrophys. and Space Phys. 7, 183.

Cherepashchuk, A.M. 1990, Astron. Zh. 67, .. (= Sov. Astron. .., ..).

Cherepashchuk, A.M. and Rustamov, D.N. 1990, Astrophys. Space Sci. 167, 281.

Cherepashchuk, A.M., Eaton, J.A., and Khaliullin, Kh.F. 1984, Astrophys. J. 281, 774.

Conti, P.S. and Vacca, W.D. 1990, Astron. J. 100, 431.

De Greve, J.P. and Doom, C. 1988, Astron. Astrophys. Suppl. 74, 325.

Doom, C. 1987, Astron. Astrophys. 182, L43.

Drissen, L., Moffat, A.F.J., Bastein, P., Lamontagne, R., and Tapia, S. 1986a, Astrophys. J. 306, 215.

Drissen, L., Lamontagne, R., Moffat, A.F.J., Bastein, P., and Seguin, M. 1986b, Astrophys. J. 304, 188.

Drissen, L., Robert, C., Lamontagne, R., Moffat, A.F.J., and St.-Louis, N. 1989, Astrophys. J. 343, 426.

Eaton, J.A., Cherepashchuk, A.M., and Khaliullin, Kh.F. 1982, in: Y. Kondo, J.M. Mead and R.D. Chapman (eds.), Advances in Ultraviolet Astronomy: Four Years of IUE Research, NASA CP-2238, p. 542.

Eaton, J.A., Cherepashchuk, A.M., and Khaliullin, Kh.F. 1985a, Astrophys. J. 297, 266.

Eaton, J.A., Cherepashchuk, A.M., and Khaliullin, Kh.F. 1985b, Astrophys. J. 296, 222.

van Genderen, A.M. and van der Hucht, K.A. 1986, Astron. Astrophys. 162, 109.

van Genderen, A.M., van der Hucht, K.A., and Steemers, W.J.G. 1987, Astron. Astrophys. $185,131$.

van Genderen, A.M., van der Hucht, K.A., and Larsen, I. 1989, Astron. Astrophys. 229, 123.

Gonzalez, J. and Rosado, M. 1984, Astron. Astrophys. 134, L21.

Gosset, E. and Vreux, J.M. 1987, Astron. Astrophys. 178, 153.

Hamann, W.R., Schmutz, W., and Wessolowski, U. 1988, Astron. Astrophys. 194, 190.

Hamann, W.R., Wessolowski, U., Schwarz, E., Dunnebeil, G., and Schmutz, W. 1990, in: C.D. Garmany (ed.), Intrinsic Properties of Hot Luminous Stars, Proc. BoulderMunich Workshop, A.S.P. Conf. Series 7, p. 259.

Harvig, V. 1987, Tartu Astrofüüs. Obs. Publ. 52, 313.

Hatchett, S.P. and McCray, R. 1977, Astrophys. J. 211, 552.

van den Heuvel, E.P.J. 1976, in: P.P. Eggleton et al. (eds.), Structure and Evolution of Close Binary Systems (Dordrecht: Reidel), p. 35.

van den Heuvel, E.P.J. 1981, Vistas in Astronomy 25, 95.

Hidayat, B., Admiranto, A.G., and van der Hucht, K.A. 1984, Astrophys. Space Sci. 99, 175.

Hillier, D.J. 1987, Astrophys. J. Suppl. 63, 965.

Hogg, D.E. 1989, Astron. J. 98, 282.

Howarth, I.D. and Phillips, A.P. 1986, Monthly Notices Roy. Astron. Soc. 222, 809.

van der Hucht, K.A. 1990, A Bibliography of Wolf-Rayet Literature 1980 - 1990 (preprint). van der Hucht, K.A., Hidayat, B., Admiranto, A.G., Supelli, K.R., and Doom, C. 1988, Astron. Astrophys. 199, 217.

van der Hucht, K.A., van Genderen, A.M., and Bakker, P.R. 1990, Astron. Astrophys. 228, 108. 
Hutchings, J.B. and Massey, P. 1983, PASP 95, 151.

Khaliullin, Kh.F. and Cherepashchuk, A.M. 1976, Astron. Zh. 53, 327 (= Sov. Astron. 20, 186).

Khaliullin, Kh.F., Khaliullina, A.I. and Cherepashchuk, A.M. 1984, Pis'ma Astron. Zh. 10, 600 (= Sov. Astron. Letters 10, 250).

Kartasheva, T.A. and Snezko, L.I. 1985, Astron. Zh. 62, 751 (= Sov. Astron. 29, 440).

Koenigsberger, G. 1988, Revista Mexicana Astron. Astrof. 16, 75.

Koenigsberger, G. and Auer, L.H. 1987, PASP 99, 1080.

Koenigsberger, G., Moffat, A.F.J., and Auer, L.H. 1987, Astrophys. J. 322, L41.

Lamontagne, R. and Moffat, A.F.J. 1987, Astron. J. 94, 1008.

Lamontagne, R., Moffat, A.F.J., and Lamarre, A. 1986, Astron. J. 91, 925.

Leung, K.C., Moffat, A.F.J., and Seggewiss, W. 1983, Astrophys. J. 265, 961.

Lipunova, N.A. 1982, Pis'ma Astron. Zh. 8, 242 (= Sov. Astron. Letters 8, 128).

Lipunova, N.A. and Cherepashchuk, A.M. 1980, Astron. Zh. 57, 1033 (= Sov. Astron. 6, 193).

Lipunova, N.A. and Cherepashchuk, A.M. 1982, Astron. Zh. 59, 944 (=Sov. Astron. 26, 569 ).

Marchenko, S.V. 1988, Kinematics and Physics of Celestial Bodies 4, 25.

Margon, B. 1984, Ann. Rev. Astron. Astrophys. 22, 507.

Monderen, P., de Loore, C.W.H., van der Hucht, K.A., and van Genderen, A.M. 1988, Astron. Astrophys. 195, 179.

Moffat, A.F.J. 1982, in: C. de Loore and A.J. Willis (eds.), Wolf-Rayet Stars: Observations, Physics, Evolution (Dordrecht: Reidel), p. 263.

Moffat, A.F.J. 1988a, Astrophys. J. 330, 766.

Moffat, A.F.J. 1988b, in: Polarized Radiation of CS Origin (Vatican), p. 607.

Moffat, A.F.J. 1989, Astrophys. J. 347, 373.

Moffat, A.F.J., Firmani, C. McLean, I.S., and Seggewiss, W. 1982, in: C. de Loore and A.J. Willis (eds.), Wolf-Rayet Stars: Observations, Physics, Evolution (Dordrecht: Reidel), p. 577.

Moffat, A.F.J. and Shara, M.M. 1986, Astron. J. 92, 952.

Moffat, A.F.J., Lamontagne, R., Shara, M.M., and McAlister, H.A. 1986, Astron. J. 91, 1392.

Moffat, A.F.J., Drissen, L., Lamontagne, R., and Robert, C. 1988, Astrophys. J. 334, 1038.

Moffat, A.F.J., Drissen, L., Robert, C., Lamontagne, R., Coziol, R., Mousseau, N., Niemela, V.P., Cerruti, M.A., Seggewiss, W., and van Weeren, N. 1990, Astrophys. J. 350, 767.

Nichols-Bohlin, J. and Fesen, F.A. 1986, Astron. J. 91, 925.

Pauldrach, A., Puls, J., Hummer, D.G., and Kudritzki, R.P. 1985, Astron. Astrophys. 148, L1.

Poe, C.H., Friend, D.B., and Cassinelli, J.P. 1989, Astrophys. J. 337, 888.

Pollock, A.M.T. 1987, Astrophys. J. 320, 283.

Prilutsky, O.F. and Usov, V.V. 1976, Astron. Zh. 53, 6.

Robert, C. and Moffat, A.F.J. 1989, Astrophys. J. 343, 902.

Robert, C., Moffat, A.F.J., Bastein, P., St.-Louis, N., and Drissen, L. 1989, Astrophys. J. 347, 1034.

Rudy, R. and Kemp, K. 1978, Astrophys. J. 221, 200.

Rustamov, D.N. and Cherepashchuk, A.M. 1986, Pis'ma Astron. Zh. 12, 373. (= Sov. Astron. Letters 12, 155).

Schulte-Ladbeck, R.E. and van der Hucht, K.A. 1989, Astrophys. J. 337, 872.

Shore, S.N. and Brown, D.N. 1988, Astrophys. J. 334, 1021. 
Shylaja, B.C. 1986, J. Astrophys. Astron. 7, 171.

Smith, L.F., Maeder, A. 1989, Astron. Astrophys. $211,71$.

Smith, L.J., Willis, A.J., Garmany, C.D., and Conti, P.S. 1986, in: E.J. Rolfe (ed.), NewInsight in Astrophysics: 8 Years of Astronomy with IUE, ESA SP-263, p. 389.

Stevens, I.R. and Willis, A.J. 1988, Monthly Notices Roy. Astron. Soc. 234, 783.

St.-Louis, N., Moffat, A.F.J., Drissen, L., Bastein, P., Robert, C. 1988, Astrophys. J. 330, 286.

St.-Louis, N., Smith, L.J., Stevens, L.R., Willis, A.J., Garmany, C.D., and Conti, P.S. 1989, Astron. Astrophys. 226, 249.

Stickland, D.J., Bromage, G.E., Budding, E., Burton, W.M., Howarth, I.D., Jameson, R., Sherrington, M.R., and Willis, A.J. 1984, Astron. Astrophys. 134, 45.

Tutukov, A.V. and Yungelson, L.R. 1973, Nauchn. Inform. Astrosov. Akad. of Sci. USSR 27, 58.

Tutukov, A.V. and Yungelson, L.R. 1985, Astron. Zh. 62, 604, (= Sov. Astron. 29, 352).

Underhill, A.B. and Fahey, R.P. 1987, Astrophys. J. 313, 358.

Underhill, A.B., Yang, S., and Hill, G.M. 1988, PASP 100, 1256.

Underhill, A.B. Gilroy, K.K., and Hill, G.M. 1990, Astrophys. J. 351, 651.

Vanbeveren, D. 1988, Astron. Astrophys. 189, 109.

Vreux, J.M. 1985, PASP 95, 274.

Vreux, J.M. 1987, in: H. Lamers \& C. de Loore (eds.), Instabilities in Luminous Early Type Stars, Proc. Workshop in Honour of Cornelis de Jager (Dordrecht: Reidel), p. 81.

Willis, A.J. and Garmany, C.D. 1987, in: H. Lamers \& C. de Loore (eds.), Instabilities in Luminous Early Type Stars, Proc. Workshop in Honour of Cornelis de Jager (Dordrecht: Reidel), p. 157.

Willis, A.J., Howarth, I.D., Conti, P.S., and Garmany, C.D. 1986, in: A.J. Willis et al. (eds.), Luminous Stars and Associations in Galaxies, Proc. IAU Symp. 116, (Dordrecht: Reidel), p. 259.

Zilayev, B.E. and Marchenko, S.V. 1989, Tartu Astrofüüs. Obs. Teated 89, 139. 


\section{DISCUSSION}

Vanbeveren: Your conclusion concerning the WR+c systems is very interesting. In 1988 I showed that the fraction of WR+c systems expected from the theory of close binary evolution may be lower than $5 \%$ of the total WR population, a result which was confirmed by Meurs and van den Heuvel (1989). As there are about 40 WR stars within $2.5 k p c$ from the sun, one then expects at most 2 WR stars with a compact companion.

Cherepashchuk: Yes, I agree. From our statistical investigation, only a small part of the suspected WR+c binary systems may be real binaries.

Moffat: I would add a comment myself here. Niemela has observed the second best candidate for a compact companion claimed by myself and others (Drissen et al.) to be a black hole, and the evidence has been weakened because it is not a run-away object problably as we once thought. This is in press, I think.

Massey: As a follow-up to Vanbeveren's question, let me note that many of the ' $W R+c$ ' orbits get an "f" rating in Batten's 8th catalogue (f: suspicious or no orbit). I think that when the semi-amplitude is very small, careful independent observations may be needed to establish whether RV variations are real and periodic.

Cherepashchuk: I agree with you. For the WR+c binaries interpretation of small amplitude radial velocity curves as orbital motions of the components and periodicity of spectral and photometric variability must be checked very carefully.

Underhill: Very often with WR components the semi-amplitude (K) is different for different lines. The question arises which line represents the motion of the center of mass? See the discussion by Underhill et al. (1990) for the case of CQ Cep.

Cherepashchuk: For the determination of the masses of WR stars it is necessary to use the emission lines with highest ionisation potential. In this case we can hope that for the binary systems with not very short periods the mass determination for WR components is satisfactory.

Koenigsberger: Is the IR eclipse in V444 Cyg symmetrical? Could it be due to the windwind collision region?

Cherepashchuk: The IR eclipse in V444 Cyg is quite symmetrical. But the IR eclipse has much more width and depth than optical ones. It is hard to understand in the model of a continuous WR wind. I suggest that a WR wind consists of many dense small blobs of matter. That allows to understand such a great dimension of the WR envelope in the IR range. The relationship between the shape of the secondary eclipse in the IR range for V444 Cyg and the shock wave around the 06 star needs further investigation.

Shylaja: I also opine that the radial velocity curves cause problems and cannot be generalized as just mentioned by Underhill. Which particular line did you choose in your amplitude vs. other parameters graphs?

Cherepashchuk: The autors quoted have used $H e I I, N I V, N V, C I I I, C I V$ emission lines. The radial velocity curves for some $W R+c$ binaries do not correspond to the true orbital motion. You are right. So for WR+c binary systems the interpretation of radial velocity curves as orbital motion should be very careful. For checking it, we have analyzed the correlations between the values of $K$ and other characteristics of supposed WR+c binaries. 


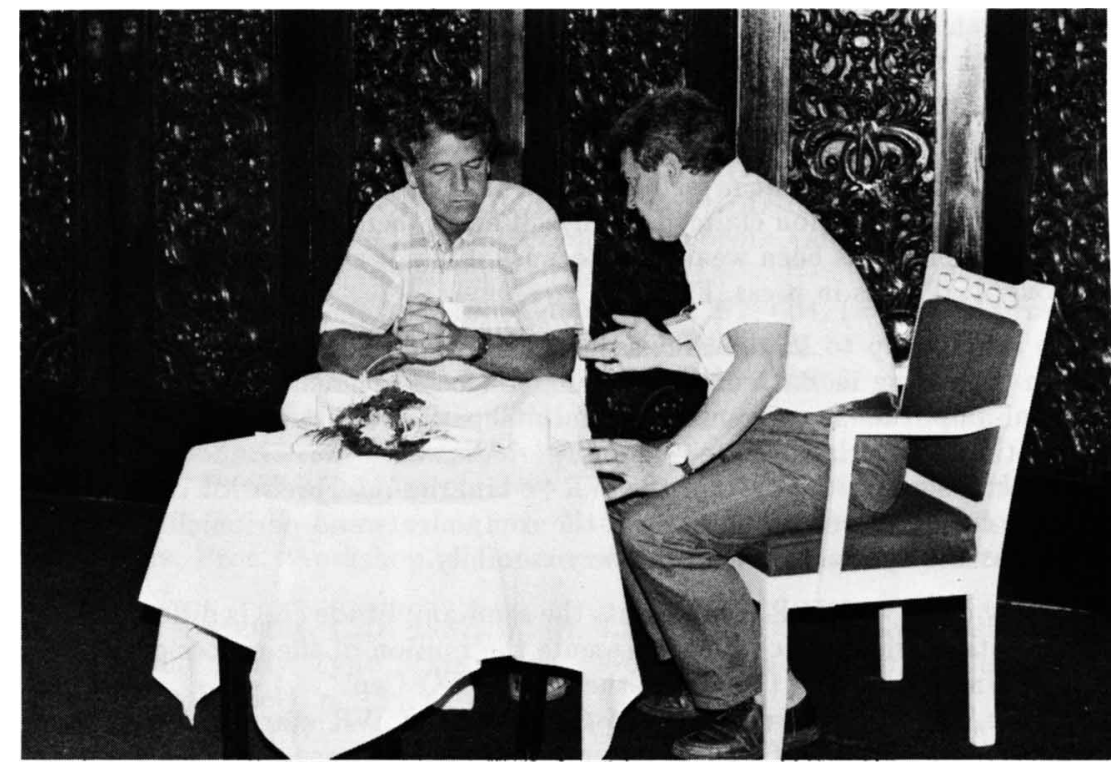

Tony Moffat, Anatol Cherepashchuk 